\title{
Morel-lavallée lesions: our treatment experience
}

\section{Introduction}

Morel-Lavallée lesions are posttraumatic soft tissue closed degloving injuries in which the subcutaneous tissue is torn away from the underlying fascia, creating a cavity filled with hematoma and liquefied fat. Morel-Lavallee syndrome or lesion was first described by a French surgeon, Victor Morel Lavallee, in $1863 .{ }^{1}$ It is also known as Morel-Lavallee seroma, post-traumatic soft tissue cyst, post-traumatic extravasations, or Morel-Lavallee effusion. ${ }^{2}$

\section{Case report}

We report five cases of extensive Morel-Lavallee lesion involving the thigh. Four males and a woman main age 48, 6 years old (Male: 60, 40, 55, 38 - Female: 50 y.o.) were admitted to the hospital for progressive soft swelling over the thigh regions. Three of them had a history of a previous admission in a hospital for road traffic accident almost 3-5 months before; with abrasion over the flank two of them had an abrasion over the thigh caused of sheep's hits, 1month before with a superficial abrasion seen on the middle of the thigh. On examination, there was tenderness and a fluctuant swelling on the lateral side of the thigh (Figure 1).

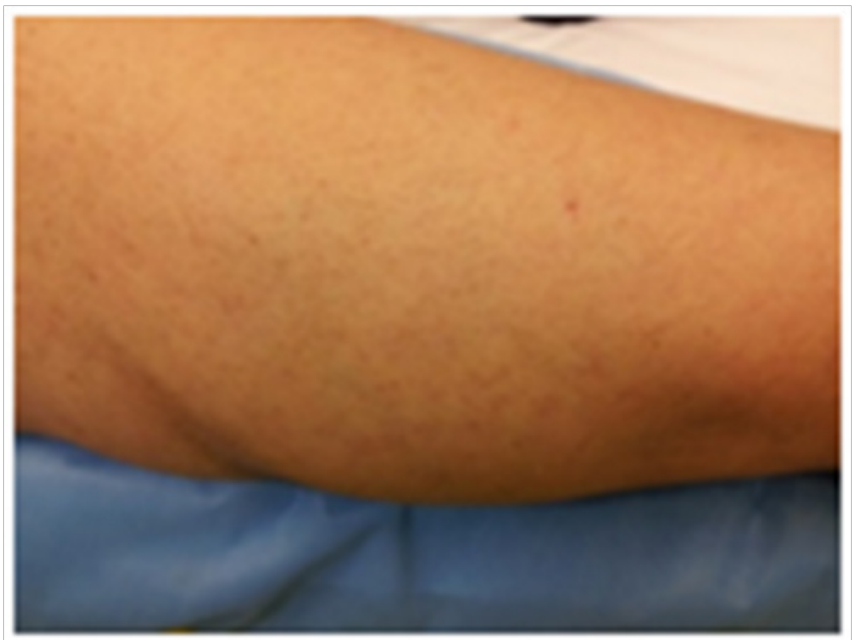

Figure I lateral side of the thigh showing tenderness and a fluctuant swelling.

\section{Diagnostic imaging examination}

The MRI showed a large subcutaneous collection that was slightly hyper intense on T1-weighted and hyper intense on T2W sequences and which was seen to extend from the upper thigh to the knee on the lateral aspect. Non-enhancing fat lobules and debris were seen within the collection (Figure 2). Blood investigations were unremarkable with no evidence of infection. Pelvic and left femur radiographs revealed no underlying fractures.

\section{Treatment}

A percutaneous drainage, debridement and irrigation were done (Figure 3). 1-1.5 liters of darkish brown fluid was drained from the
Volume 2 Issue 5 - 2015

\author{
Athanasios Alexandris, Christos \\ Alexandropoulos, Vaios Goulas, Stefanos \\ Tsourvakas \\ Department of Orthopedics, Greece
}

Correspondence: Athanasios Alexandris, Department of Orthopedics, General Hospital of Trikala, Karditsis 5 I Trikala 42100, Greece, Tel 00306947445950,Email thalexandris@yahoo.gr

Received: March 26, 2015 | Published: May 19, 2015

subcutaneous fat plane above the muscle fascia. Areas of necrotic fat were also seen. The underlying soft tissue was not infected. No bacterial organism or growth was cultured from the fluid. Sanction drainage and compression dressing was applied for 24 hours, after that a pair of hip graduated compression stockings (Figure 4) are applied and the patient was discharged without any complication the next day with instructions for wearing them all day for one month. The first follow up was 10 days after for cutting the stitches and the second 30 days after without any complications. A year after no relapse was observed.
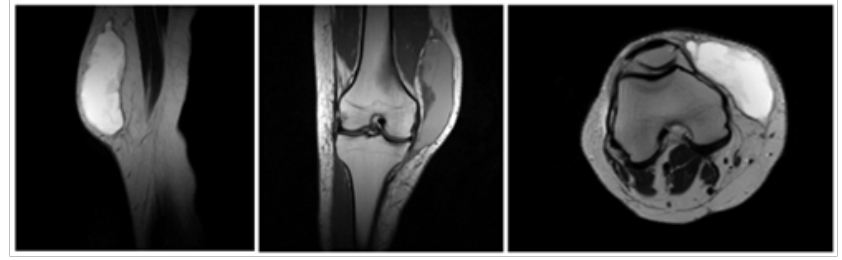

Figure 2 Non-enhancing fat lobules and debris.

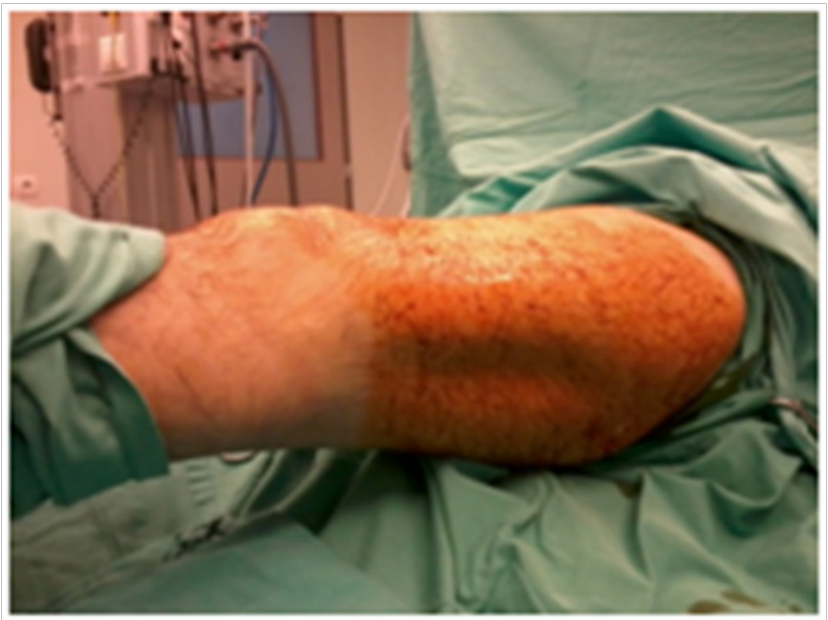

Figure 3 Showing percutaneous drainage, debridement and irrigation. 


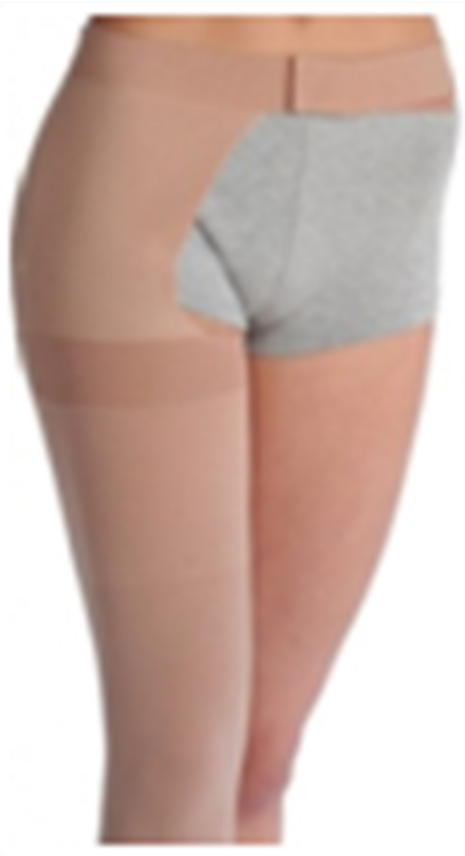

Figure 4 Showing no relapse.

\section{Discussion}

Morel-Lavallee lesions usually present within a few hours to months or years post initial trauma. They are frequently associated with underlying fractures or not. These lesions are most often unilateral. ${ }^{1,2}$ The initial injury represents a shearing of subcutaneous tissues away from the underlying fascia. The disrupted capillaries may continuously drain into the perifascial plane, filling up the virtual cavity with blood, lymph, and debris. Subsequent inflammatory reaction may lead to a peripheral capsule formation, which may account for the self-perpetuation and occasional slow growth of the process. $^{2}$ The collection may then spontaneously resolve, or become persistent with encapsulation. Patients usually present with complaints of pain, swelling, and stiffness. On clinical examination, patients often have a soft fluctuant area of contour deformity, with or without skin discoloration. ${ }^{3}$ The differential diagnosis for MLL includes subcutaneous hematoma, hemangioma, fat necrosis, and soft tissue sarcoma.

The history of trauma, characteristic location, and MRI features may contribute to a correct diagnosis., ${ }^{2,3}$ The treatment depends on the duration, size, and presence of a capsule in the lesion. Small acute lesions that have not developed a capsule can be treated conservatively by application of compression bandage. However, those that persist and have capsule formation may require more aggressive treatment. These lesions can be managed with early percutaneous drainage, debridement, irrigation, and suction drainage. ${ }^{4}$ These lesions also, to be resolved permanently needed compression bandage for long time. Very comfortable for the patients are the hip graduated compression stockings for prevention of thromboembolism..$^{5-11}$

\section{Conclusion}

They can use them easily, wearing them during day and night, under their clothes without feeling discomfort able; taking them off while taking a shower and put them easily back. The graduated compression that the hip stockings perform helps the skin to agglutinate on the underline fascia and prevents the relapses.

\section{References}

1. Hudson DA, Knottenbelt JD, Krige JE. Closed degloving injuries: results following conservative surgery. Plast Reconstr Surg. 1992;89(5):853855 .

2. Hak DJ, Olson SA, Matta JM. Diagnosis and management of closed internal degloving injuries associated with pelvic and acetabular fractures: the Morel-Lavallée lesion. J Trauma. 1997;42(6):1046-1051.

3. Li H, Zhang F, Lei G. Morel-Lavallee lesion Chinese Medical Journal. 2014;127(7):1351-1356.

4. Al Hetmi T, Hammouda A, Makki A. Morel-lavalle syndrome. Qatar Med J. 2006;15:40-42.

5. Letournel E JR. Fractures of the Acetabulum. (2nd edn), SpringerVerlag, Berlin, Germany, 1993.

6. Guerrero LA, Therattil MR. Post-traumatic morel-lavallée seroma in young female pedestrian struck by a moving bus: A case report. PM\&R. 2012;4(10):S259.

7. Lin HL, Lee WC, Kuo LC, et al. Closed internal degloving injury with conservative treatment. Am J Emerg Med. 2008;26(2):254-255.

8. Scaranelo AM, Davanco RA. Pseudocyst formation after abdominal liposuction-extravasations of Morel-Lavallée on MR images. Br J Plast Surg. 2005;58(6):849-851.

9. Goodman BS, Smith MT, Mallempati S, et al. A comparison of ultrasound and magnetic resonance imaging findings of a Morel-Lavallee lesion of the knee. PM R. 2013;5(1):70-73.

10. Moriarty JM, Borrero CG, Kavanagh EC. A rare cause of calf swelling: the Morel-Lavallee lesion. Ir J Med Sci. 2011;180(1):265-268.

11. Lamyman MJ, Baden JM, Reid CD. The diagnosis and management of an expanding post-traumatic soft tissue cyst of the hip and groin. J Plast Reconstr Aesthet Surg. 2009;62(10):394-397. 